\title{
TRAUMATIC INTRAMURAL HAEMATOMA OF THE PROXIMAL JEJUNAL LOOP*
}

\author{
BY \\ F. H. ROBARTS \\ From the Royal Hospital for Sick Children, Edinburgh
}

That injury to the abdominal viscera can result from a blow by a blunt object on the abdominal wall has been recognized from the earliest days of medical knowledge; and that such a blow can leave no visible trace upon the skin of the abdominal wall was also known at that time. For, in the third century B.C., Aristotle is reputed by Morgagni (1761) (Epistola, quoted by Poer and Woliver) to have written that 'A slight blow will cause rupture of the intestine without injury to the skin'.

Poer and Woliver (1942) reviewed the literature of injuries of the intestines and mesenteries due to non-penetrating abdominal trauma and found numerous references in the literature from 1875 onwards, thus proving the continual occurrence of such injuries. These authors stressed the importance of early diagnosis, for all the cases reported up to that time were associated with rupture of the intestines or with injuries to the mesenteries. A mortality of $35 \%$ was found in those cases which were diagnosed and treated by operation at an early stage. For those in which there was a delay of over 12 hours the mortality rose to $70 \%$.

Counseller and McCormack (1935) also published a survey of subcutaneous rupture of the intestine. Seventy-seven of the 1,183 cases found in the literature up to that time occurred in children under the age of 12 years. At all ages the jejunum was most often involved (447 occasions) and the ileum slightly less often (412 cases). In 29 instances the site of injury was either not noted or no perforation was found at laparotomy. In this group there may have occurred cases of haematoma of the bowel wall not considered worthy of note or record.

In yet a third and even earlier survey (Vance, 1923), it was stated that the jejunum and ileum were about equally involved in injuries of the subcutaneous type, and that the most frequently affected sites were those of natural fixation in the duodenojejunal area and the ileo-caecal junction.

It was not until 1952 (Zabin, 1952) that

* A paper read at the meeting of the British Association of Paediatric Surgeons held in Edinburgh in June, 1957. haematoma of the bowel wall as the cause of an acute surgical condition was cited. A boy of 4 years was struck in the left upper abdomen by the handlebar of a bicycle, and within six hours was complaining of abdominal pain. This symptom only became severe enough to warrant his admission to hospital two days later. A small abrasion of the abdominal wall was discerned at the site of impact with the handlebar. The significance of the injury was not appreciated and a pre-operative diagnosis of acute appendicitis was made. At operation there was a large amount of blood-tinged free fluid in the peritoneal cavity. A lower loop of jejunum, 14 in. long, lying in the left hypochondrium was found to be the site of a large haematoma. Resection was performed. The haematoma was found to lie under the circular muscle layer. The child recovered.

In 1954, Lampert, Goodfellow and Wachowski reported the case of a lad of 15 years who received a kick in the abdomen during a game of basketball. Although complaining of abdominal pain he was not admitted to hospital until two days later and there a further two days elapsed while obstructive symptoms and signs became more marked and the presence of an urgent abdominal condition quite definite. At operation a subserosal haematoma immediately distal to the duodeno-jejunal junction was found. The clot was evacuated from the bowel wall and recovery ensued.

During the last 10 years in the Royal Hospital for Sick Children, Edinburgh, four cases of jejunal injury without external evidence of violence have been admitted. Two of these, in girls of 8 and 5 years respectively, were associated with perforation of the gut; the first of the second loop of jejunum and the other of a lower loop. The first child had fallen on the high step of a barn; the second was squeezed by the rear wheel of a reversing G.P.O. mail van. In each case operation was performed within six hours of injury and recovery of both ensued.

The other two cases are of greater interest. 
Case Reports

Case 1. On April 29, 1947, E.H., a boy of 9 years, fell off a tricycle which he was probably far too big to be riding. The handlebars struck him in the left upper abdomen. He immediately complained of pain and a bruise developed at the point of impact. Two days later, having carried on more or less normally in the meantime, he came home from school looking very pale and shortly afterwards he vomited several times. $\mathrm{He}$ complained of pain in the abdomen on vomiting and he continued to vomit everything he drank, the vomits soon becoming dark green. His bowels, however, continued to act, having last done so on the day before his admission five days after injury.

On examination of the abdomen, the respiratory excursion was moderately good. There was no acute tenderness on palpation but some guarding was appreciated in the upper left zone. The result of the examination was rather indefinite, but operative exploration was arranged after gastric aspiration had been instituted and his dehydration and shocked state improved by intravenous plasma and saline infusion.

At operation, through an upper right paramedian incision, no free fluid or blood was found in the peritoneal cavity. All the loops of small intestine which were immediately visible were noted to be tightly contracted and collapsed. A large tense, grossly distended loop of jejunum was felt in the upper left quadrant. This was found to be the first loop of jejunum. It was black, distended to approximately the size of a 'black pudding', and had a sharp demarcation between bowel of normal colour. The serous coat had not lost its sheen and the bowel was felt grossly thick to the touch. In delivering the loop, a small tear of the serous coat resulted and there was an immediate escape of blood and blood clot, but no faecal matter was evident. The glands in the mesentery of this loop were enlarged and there was also some formation of haematoma in the mesentery, in which the blood vessels were felt to pulsate normally. The affected loop was resected. This left insufficient normal gut to make an anastomosis possible. The short upper jejunal loop was closed and invaginated with some difficulty owing to its proximity to the duodeno-jejunal flexure. The lower loop of bowel was also closed and a posterior gastro-jejunostomy performed between it and the stomach.

Immediate examination of the specimen showed a portion of jejunum approximately 8 in. long. It was grossly distended. Normal bowel ended abruptly both above and below in purplish-black discoloration of the gut. The gut wall was thickened and felt solid. The specimen was cut after fixation in formalin and it was found that the lesion consisted of haemorrhage into the subserous coats of the gut wall. The large amount of blood had clotted and had stripped the mucosa and so compressed it that only a minute lumen remained. More detailed examination showed that the wall of the bowel had been disrupted by haemorrhage chiefly in the submucosal area, a large mass of recently clotted blood separating the mucosa from the muscular coats (Fig. 1). The mucous membrane and the muscularis mucosae were remarkably normal in appearance, there being a little congestion of the villi. The effused blood was mainly in the outer part of the submucosa. Some veins in this part showed recent thrombosis. The circular muscle layer was infiltrated by the blood so that the muscle fibres had been separated. The longitudinal layer was intact but much stretched so that it appeared to be very thin. In no part was there any evidence of an inflammatory reaction.

The child's immediate convalescence was complicated by persistent regurgitation of large amounts of heavily

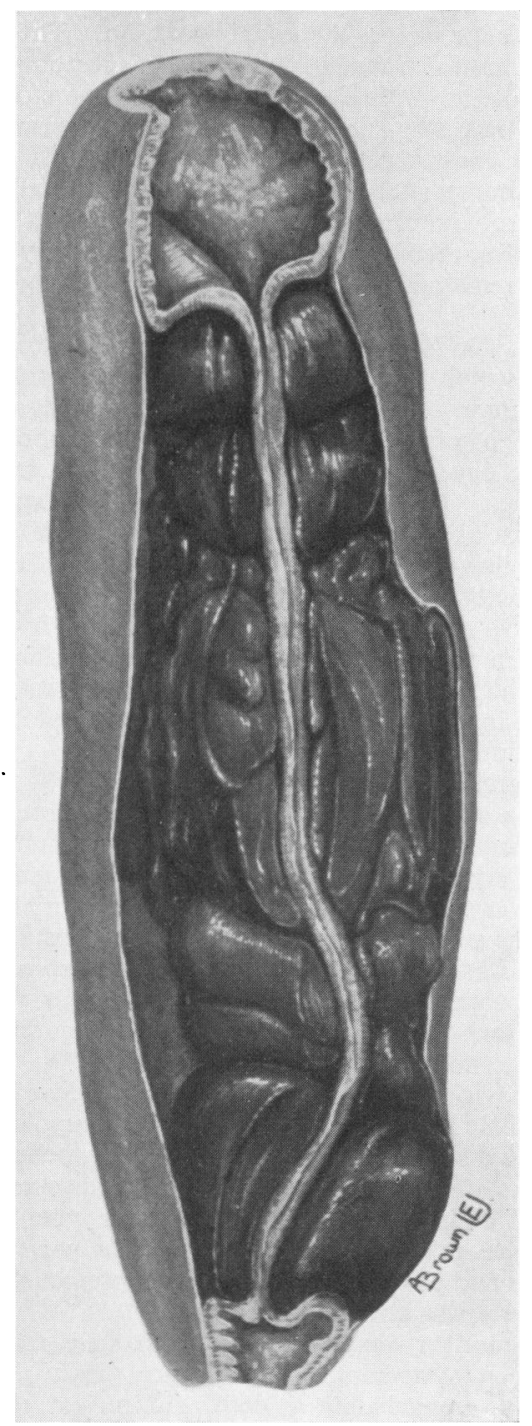

FIG. 1.-Semi-diagrammatic drawing of jejunal specimen resected in Case 1, showing intramural blood clot and compression of the bowel lumen. 
bile-stained fluid, and later he continued frequently to vomit copiously similar material. Six weeks after the first operation his readmission to hospital was necessitated by an even more severe bout of vomiting. Laparotomy was performed on the following day. The proximal jejunal stump was found to be dilated and elongated, and it was laterally anastomosed to the efferent loop of the gastro-jejunostomy.

Again episodes of vomiting occurred over the next 15 months and it was firmly thought that these attacks were of psychological origin, for repeated barium investigations showed no significant abnormality. The vomiting, however, demanded further exploration two years from the time of the initial operation. The efferent jejunal loop was traced to the gastro-jejunostomy stoma. The blind end of that loop proximal to the stoma was found to have elongated to about $15 \mathrm{~cm}$. and was dilated. A lateral anastomosis was made between this and the jejunum immediately below the duodeno-jejunal flexure. The gastro-jejunostomy was then undone.

Thereafter recovery was rapid and satisfactory and the boy has remained well.

Case 2. On November 4, 1956, W.K., a boy of 8 years, fell and struck the upper left abdomen across the back of a low chair. He fell asleep shortly afterwards and little was thought of the incident. He went to school on the following day but was sent home as he had been sick three times. He was seen by his doctor who could find no serious abdominal signs. He went to school again on the following day. Repeated vomiting and the complaint of abdominal pain caused him to be sent home again. On the third morning after injury his doctor found him to be tender over the whole abdomen and referred him to hospital. He had been in contact with a case of infective hepatitis. His bowels acted normally before admission.

On examination, he was slightly febrile $\left(99^{\circ} \mathrm{F}\right.$.) and the pulse rate was 130 per minute, respiration rate 28 per minute. He did not look ill and was found sitting up comfortably. There was no visible jaundice, the tongue was clean, and the breath odour was a little foul. There was slight tenderness under the left costal margin where it was thought that the edge of the spleen could be felt protruding to one fingerbreadth. It was also thought that the liver margin was palpable and tender. There was no sign of superficial injury.

The provisional diagnosis of an early infective hepatitis or of splenic bruising was made. Five hours later there was more distinct tenderness in the left hypochondrium. Ten hours after admission, there now being acute tenderness and definite guarding in the left hypochondrium with some diffuse tenderness in other parts of the abdomen and absence of bowel sounds previously heard, laparotomy was considered essential.

The abdomen was opened by a left transverse upper incision. On opening the peritoneal cavity, no free blood was immediately evident, but on exploring the left upper quadrant a turgid, congested and oedematous, grossly thickened proximal loop of jejunum was encountered. Close up to the duodeno-jejunal flexure an intra- mural haematoma, about 3 in. long and surrounding two-thirds of the bowel circumferentially, was present. A small perforation of the serosal coat towards the distal part of the haematoma was oozing dark blood, and in the peritoneal cavity, both above and below the left phrenicocolic ligament and largely below it, was a considerable amount of free and fluid blood. The spleen was of normal size and uninjured. There was considerable oedema in the anterior wall of the lesser sac, especially just below the stomach, but no other injury could be found. Above the jejunal haematoma the normal bowel was insufficient to permit easy anastomosis after resection for it was oedematous and friable. In order to control the bleeding and overcome the intestinal obstruction caused by compression of the gut lumen, the serosa was incised. Adherent and organizing blood clot was with little difficulty evacuated. The underlying muscle coats of the bowel appeared to be healthy but no major bleeding point could be found. The bulk of the haematoma was thus reduced and the thickness of the bowel in this area approximated to the normal. Gaseous bowel content could now be readily expressed proximally through the abnormal segment. The serosal defect was repaired partly by suture and partly by a free omental graft. Intragastric suction was discontinued on the third post-operative day and intravenous infusion on the fourth day. Thereafter recovery was uneventful and a barium meal on the thirteenth day revealed a free flow through the duodenum and jejunum. He was discharged on that day and has remained well.

\section{Discussion}

With the relation of the details of these two cases and with the knowledge of one of the two cases already reported in the literature and mentioned briefly above, it is obvious that intramural haematoma of the proximal jejunal loop is no bizarre injury although it is rare. It is a condition which presents several typical features.

It is a child or an adolescent who sustains this injury; it may be only fortuitous that the male alone has been thus afflicted. In him at this age the abdominal musculature has not developed its maximum strength and thickness, the lower rib cage is free and on that account more flexible, and the antero-posterior depth of the abdominal cavity is smaller than in the adult. At the time of impact in the upper left abdomen the musculature is further weakened and caught off guard by the forward flexion of the trunk. It is well known that the fixity of the bowel in this situation, as also in the ileo-caecal region, renders it more vulnerable to injury by sudden and deep violence. The vertical orientation of this loop and its situation to the left and behind the central prominence of the vertebral bodies and the posterior relation of the retroperitoneal musculature have the effect of cushioning the bowel at impact. Similar factors operated in 
a case of haematoma of the wall of the ascending colon, although in that case the condition only came to light some months later with organization of the clot and the production of a palpable tumour and a space-filling lesion on examination by a barium enema (Kratzer and Dixon, 1951). Less fixed loops of small bowel are more vulnerable to such injury when they lie transversely and as they cross the anterior prominence of the vertebral bodies. In these circumstances perforation of the affected loop appears to be more probable although, as mentioned above, an acute intramural haematoma may result (Zabin, 1952).

The pathological effects of this injury are not immediate and, as the bleeding into the layers of the bowel wall proceeds, increasing disturbance to bowel function develops. Intraperitoneal bleeding, if it occurs, would appear to be a later effect. If such bleeding were an early feature, it would seem probable that the escape of blood from the bowel wall would limit the degree of stripping of the muscle layers and diminish the compression effect upon the bowel lumen and retard or diminish the resulting intestinal obstruction. This obstruction is not entirely the result of mechanical compression but is also and perhaps even to a major degree the result of the disruption and infiltration of the muscular coats of the bowel wall. Such intramural bleeding has been reported as occurring in the small intestine in association with duplication, ulceration within which may penetrate a small but significant blood vessel (White and Wollenman, 1956). A similar case, presenting with intestinal obstruction, has been encountered personally.

The clinical course after injury presents typical features. After the momentary effects of the blow in the region of the solar plexus, a latent and quiescent period of some hours, and usually of a day or two, ensues. Increasingly severe upper left abdominal pain associated with recurring vomiting follows. Gradually the indeterminate physical signs become more definite. Earlier recognition of the urgent condition seems more probable where there is eventually an associated intraperitoneal leakage of blood.
At laparotomy which, in the circumstances of the typical injury and in the presence of the developing acute abdominal signs, is desirable and probably inevitable, this lesion is encountered in one of the more inaccessible reaches of the abdomen. The lapse of time which has often occurred since injury has permitted the development of a considerable amount of reactionary oedema and an inflammatory response. Resection and reconstitution by anastomosis may appeal as a definitive form of treatment, but in this situation and in these circumstances, these procedures to be safe are difficult. The intramural bleeding which occurs in Henoch's purpura, with the resulting obstructive state, is often successfully countered by conservative means. This suggests that a less radical approach to the present problem is justifiable. That this is so is borne out by the second case reported here and by the other typical case described by Lampert, Goodfellow and Wachowski (1954). Intramural haemorrhage in association with duplication will always necessitate resection.

\section{Summary}

The literature concerning traumatic lesions of the intestines caused by non-penetrating blunt blows is reviewed with especial reference to the jejunum and intramural haematoma of its proximal loop.

Two cases of traumatic intramural haematoma of the proximal jejunal loop are described.

The aetiological, pathological and clinical features of the condition are detailed.

The treatment of this and similar lesions is discussed.

I have to thank Mr. J. J. Mason Brown for permission to quote fully the details of Case 1 .

\section{REFERENCES}

Counseller, V. S. and McCormack, C. J. (1935). Ann. Surg., 102, 365 .

Kratzer, G. L. and Dixon, C. F. (1951). Proc. Mayo Clin., 26, 18. Lampert, E. G., Goodfellow, J. G. and Wachowski, T. J. (1954). Ann. Surg., 140, 768.

Morgagni, J. B. (1761). Epistola, quoted by Poer and Woliver. Poer, D. H. and Woliver, E. (1942). J. Amer. med. Ass., 118, 11. Vance, B. M. (1923). Arch. Surg. (Chicago), 7, 197.

White, R. J. and Wollenman, O. J. (1956). Ann. Surg., 143, 720 Zabin, A. (1952). N.Y. St. J. Med., 52, 93. 